\title{
Modelling the optical response of gold nanoparticles $\dagger$
}

\author{
Viktor Myroshnychenko, ${ }^{a}$ Jessica Rodríguez-Fernández, ${ }^{b}$ Isabel Pastoriza-Santos, ${ }^{b}$ \\ Alison M. Funston, ${ }^{c}$ Carolina Novo, ${ }^{c}$ Paul Mulvaney, ${ }^{c}$ Luis M. Liz-Marzán ${ }^{b}$ and \\ F. Javier García de Abajo*a
}

\author{
Received 27th June 2008 \\ First published as an Advance Article on the web 23rd July 2008 \\ DOI: $10.1039 /$ b711486a
}

\begin{abstract}
This tutorial review presents an overview of theoretical methods for predicting and understanding the optical response of gold nanoparticles. A critical comparison is provided, assisting the reader in making a rational choice for each particular problem, while analytical models provide insights into the effects of retardation in large particles and non-locality in small particles. Far- and near-field spectra are discussed, and the relevance of the latter in surface-enhanced Raman spectroscopy and electron energy-loss spectroscopy is emphasized.
\end{abstract}

\section{Introduction}

Gold nanoparticles have been used as colourants for almost 2000 years, principally in stained glass windows, ceramics and enamel pottery, but also as a purple dye in clothes. ${ }^{1}$ Throughout this long period, dating back to Roman times, there was no understanding of the underlying mechanisms which give rise to the spectacular colours of small metal particles. Maxwell-Garnett explained many of the scattering effects and colour changes in two seminal papers in 1904 and $1906,{ }^{2}$ and ultimately, the size dependent optical properties of metal spheres were quantitatively explained by Mie in $1908 .^{3}$ However, there was little interest at that time in how the shape of the nanoparticles might influence their optical properties, despite the predictions of Gans' 1912 paper on the colour of ellipsoids, ${ }^{4}$ because there were no chemical or physical methods to control the shape of small particles. It was not until several decades later that anisotropic metal nanoparticles were found during latent image

\footnotetext{
${ }^{a}$ Instituto de Óptica-CSIC, Serrano 121, 28006 Madrid, Spain. E-mail: jga@cfmac.csic.es; Fax: 34915645 557; Tel: 34653700 342

${ }^{b}$ Departamento de Química Física, and Unidad Asociada CSICUniversidade de Vigo, 36310 Vigo, Spain

'School of Chemistry, The University of Melbourne, Victoria 3010, Australia

$\dagger$ Part of a thematic issue covering the topic of gold: chemistry, materials and catalysis.
}

development in photographic emulsions, and their optical properties interpreted in terms of Gans' theory. ${ }^{5}$

On the theoretical side, the computationally demanding nature of the electromagnetic calculations needed to predict far-field scattering and extinction of non-spherical morphologies also impeded progress. Computational methods were pioneered by Purcell and Pennypacker ${ }^{6}$ using the discrete dipole approximation (DDA) to simulate more complex targets, but this did not gain widespread use until 10-15 years ago. ${ }^{7,8}$ However, the past decade has witnessed a tremendous improvement in the understanding and control of the response of small metal particles to light. ${ }^{9-12}$ This has been driven by the rapid development of many new computational tools, such as the finite difference in the time domain method ${ }^{13,14}$ (FDTD) and the boundary element method ${ }^{15,16}$ (BEM), as well as by the astounding progress in the chemical synthesis of diverse metal particles of different shapes and sizes. ${ }^{10,11}$ It has also been aided by the availability of high quality CCD spectrometers and near-field scanning techniques that allow far- and near-field spectra of single nanocrystals to be collected routinely. ${ }^{17}$

This progress has generated great expectations that there will be new applications ranging from plasmonic structures for photonic circuitry and optical communications to detection of single molecules and high throughput biological assays based upon surface enhanced Raman spectroscopy (SERS). Actually, gold nanoparticles are appealing model systems for several reasons. Firstly, gold is chemically inert and does not
Paul Mulvaney is currently an ARC Federation Fellow and Professor of Chemistry at the University of Melbourne. After a PhD from the University of Melbourne (1989), he was a research scientist at the Hahn-Meitner Institute, Berlin (1989 to 1992), and later a Humboldt Research Fellow at the Max-Planck and CAESAR Institutes. His interests include surface plasmon spectroscopy and nanoscale chemistry and physics.
Luis M. Liz-Marzán received his PhD from the University of Santiago de Compostela in 1992. He then moved to Utrecht University as a postdoctoral Research Associate. He joined the University of Vigo in 1995, where he currently is a Full Professor. His current interests include nanoparticle synthesis, colloidal composites, nanoplasmonics, and the use of metal nanoparticles as biosensors. LMLM is a Fellow of the Royal Society of Chemistry
F. Javier Garcia de Abajo received his PhD from the University of the Basque Country in 1993, where he was an Associate Professor until 1997. He was a Research Fellow at Lawrence Berkeley National Laboratory during 1997-2000 and is currently a Research Professor at the Spanish Scientific Research Council (CSIC). FJGA is a Fellow of the American Physical Society and the Optical Society of America. 
oxidize (oxide layers drastically complicate the calculation of optical properties). Secondly, this metal has a bulk plasmon resonance in the visible part of the spectrum, while for most other metals this resonance occurs in the ultraviolet (UV) region. Consequently, changes to the resonance behaviour in small gold particles lead to visible changes in colour. Finally, new methods for synthesis now enable a wide range of shapes and sizes of gold particles to be produced. ${ }^{11}$ In this context, colloid chemistry has become the tool of choice when highquality particles are required, yielding narrow size and shape distributions with finely tunable optical properties.

\subsection{Plasmons and optical response of gold particles}

The optical properties of gold nanoparticles in the visible and near-infrared (vis-NIR) domains are governed by the collective response of conduction electrons. These form an electron gas that moves away from its equilibrium position when perturbed by an external light field (see Fig. 1), thus creating induced surface polarization charges that act as a restoring force on the electron gas. This results in collective oscillatory motion of the electrons similar to the vibrations of a water surface and characterized by a dominant resonance band lying in the vis-NIR for gold: the so-called plasmon excitations, a name inherited from their initial description in terms of the oscillations of a plasma representing the conduction electron gas. $^{18}$

Plasmons produce strong effects in both the near- and farfield response of gold nanoparticles. The far-field is relevant when discussing absorption and scattering in colloidal dispersions ${ }^{19}$ or when investigating single particles using dark field microscopy (DFM). ${ }^{20,21}$ Understanding the far field is important for creating materials with tailored macroscopic properties, such as the effective dielectric function of metamaterials formed by nanoparticle aggregates, or for tuning the colour of a composite containing the particles. In contrast, near-field properties affect the surroundings of the particle within a distance smaller than or of the order of the light wavelength. The near field is accessible via scanning near-field optical microscopy $^{22}$ (SNOM) with limited spatial resolution, and more recently, it has been probed using cathodoluminescence $^{23}$ and electron energy-loss spectroscopy ${ }^{24,25}$ (EELS) performed within electron microscopes with truly nanometre spatial resolution. Near-field properties rule the optical interaction between particles placed in close proximity (e.g., in closely-packed arrays) and also the interaction with nearby

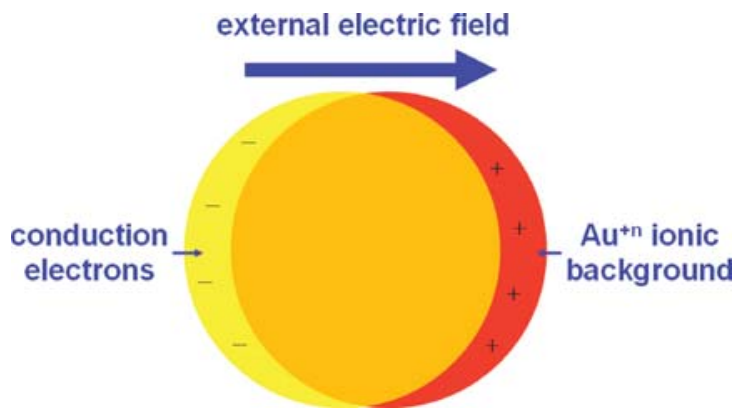

Fig. 1 Schematic representation of a dipolar plasmon oscillation in a gold nanoparticle. molecules, which is useful for sensing applications, such as SERS. $^{26}$

The far-field performance of the nanoparticles is summarized in the wavelength-dependent absorption and scattering cross sections, $\sigma_{\mathrm{abs}}$ and $\sigma_{\mathrm{sc}}$, which are defined as the effective area of a screen with which the incoming light interacts (by absorption or scattering) in a straight-ray picture. The light intensity transmitted through a dilute dispersion I (i.e., assuming no more than one photon-particle collision per photon) is

$$
I=I_{0} \exp \left[-\left(\sigma_{\mathrm{abs}}+\sigma_{\mathrm{sc}}\right) N L\right],
$$

where $I_{0}$ is the incident light intensity, $N$ is the number of particles per unit volume, and $L$ is the pathlength inside the dispersion. The remaining light intensity $\left(I-I_{0}\right)$ is lost either by absorption or by scattering of the light in all directions, and is given by the fractions $\sigma_{\mathrm{abs}} /\left(\sigma_{\mathrm{abs}}+\sigma_{\mathrm{sc}}\right)$ and $\sigma_{\mathrm{sc}} /\left(\sigma_{\mathrm{abs}}+\sigma_{\mathrm{sc}}\right)$, respectively.

The total or extinction cross section (absorption plus scattering) can be easily calculated from the far-field amplitude $f$ in the forward direction, using the optical theorem, ${ }^{27}$ as

$$
\sigma_{\mathrm{abs}}+\sigma_{\mathrm{sc}}=\frac{2 \lambda}{\sqrt{\varepsilon_{m}}} \operatorname{Im}\{\boldsymbol{f} \cdot \hat{\boldsymbol{e}} *\},
$$

where $\varepsilon_{\mathrm{m}}$ is the permittivity of the medium outside the particle, $\lambda$ is the free-space light wavelength, and $\hat{\boldsymbol{e}}$ is the incident field amplitude, normalized to unity.

Classical electromagnetic solutions provide an excellent description of the optical properties of gold nanoparticles, with gold represented by its bulk, frequency-dependent dielectric function $\varepsilon(\omega)$, as determined from optical measurements in macroscopic films ${ }^{28}$ (see Fig. 2). The dielectric function can be well approximated by a free-electron Drude model (dashed curves in Fig. 2),

$$
\varepsilon(\omega) \approx \varepsilon_{\mathrm{b}}-\frac{\omega_{\mathrm{p}}^{2}}{\omega\left(\omega+i \tau^{-1}\right)},
$$

where $\hbar \omega_{\mathrm{p}}=9 \mathrm{eV}$ is related to the density of conduction electrons $n$ via $\omega_{\mathrm{p}}=\sqrt{4 \pi e^{2} n / m}, e$ and $m$ are the electron charge and mass, $\hbar \tau^{-1}=0.05 \mathrm{eV}$ is a phenomenological electron-motion damping rate adjusted to match the imaginary part of $\varepsilon$, and $\varepsilon_{\mathrm{b}}=9$ represents the background polarization of $\mathrm{Au}$ cores.

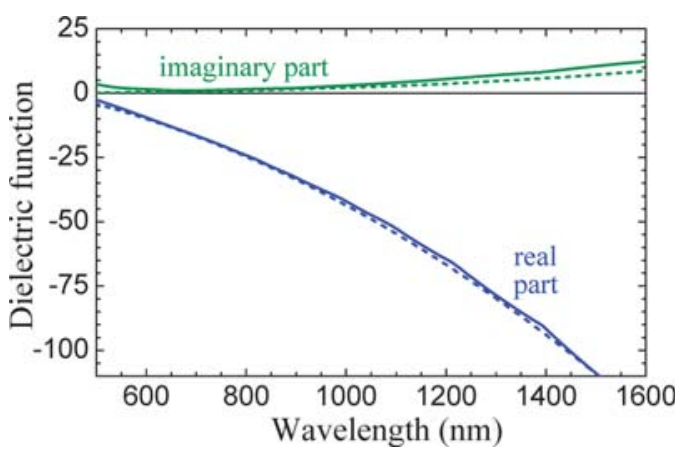

Fig. 2 Dielectric function of gold taken from ref. 28 (solid curves) and compared with the Drude model approximation of eqn (2) (dashed curves). 
The optical modelling of nanoparticles thus relies on the solution of Maxwell's equations for each specific geometry and set of illumination conditions, assuming a local $\varepsilon(\omega)$ description of the materials involved. A summary of the most established methods is presented below, including both analytical and numerical approaches, capable of dealing with isolated particles as well as particle assemblies. It should be mentioned however that the local approximation fails dramatically for particle sizes below a few nanometres, in which case finite-size effects, and more generally non-local interactions, can dominate the response, as explained in section 4 .

\section{Non-retarded description of spherical and ellipsoidal particles}

In the limit of very small particles, the electromagnetic interaction between different parts of the metal is almost instantaneous (i.e., the speed of light can be taken as infinite). Then, terms connecting electric and magnetic fields disappear from Maxwell's equations, leading to the condition that electric and magnetic fields are longitudinal $(\nabla \times \boldsymbol{E}=\nabla \times \boldsymbol{H}=0)$. The magnetic response of the particles is negligible at optical frequencies for small particles, and this leaves us only with the electric field, which can be expressed as the gradient of a scalar electric potential, $\boldsymbol{E}=-\nabla \phi$, because it is longitudinal. This electrostatic limit is best solved by inserting the field into Coulomb's law, which reduces to Poisson's equation

$$
\nabla \cdot \varepsilon(\boldsymbol{r}, \omega) \nabla \phi(\boldsymbol{r}, \omega)=0,
$$

written in frequency space and in the absence of external charges. Notice that the dielectric function depends on position (i.e., whether point $\boldsymbol{r}$ is inside or outside the particle).

The homogeneous sphere admits a textbook analytical solution in the electrostatic limit that yields the multipolar polarizability (in SI units)

$$
\alpha_{l}=3 V \varepsilon_{\mathrm{m}} \frac{l\left(\varepsilon-\varepsilon_{\mathrm{m}}\right)}{l \varepsilon+(l+1) \varepsilon_{\mathrm{m}}},
$$

where $V$ is the particle volume, $\varepsilon$ and $\varepsilon_{\mathrm{m}}$ are the permittivities of the particle and the surrounding medium, and $l$ is the orbital momentum number (e.g., $l=1$ for a dipole). This is a very useful formula for predicting the multipolar plasmon wavelength of relatively small particles, and it is the basis of the Maxwell-Garnett effective medium theory ${ }^{2,29}$ discussed in section 10.1. However, it should be used with care, because it fails for particle sizes above a few tens of nanometres due to retardation, as we show in section 3 , and it requires finite-size corrections for small radii, as explained in section 4 .

Incidentally, eqn (3) illustrates a common feature of the response of metallic nanoparticles: as the order of the plasmon modes increases, their energies evolve towards the non-retarded planar-surface plasmon given by the condition $\varepsilon=$ $-\varepsilon_{\mathrm{m}}$ (e.g., in the $l \rightarrow \infty$ limit of eqn (3)), or equivalently by $\hbar \omega=\hbar \omega_{\mathrm{p}} / \sqrt{\varepsilon_{\mathrm{b}}+1}=2.8 \mathrm{eV}$ for gold in air (see eqn (2)). In simple terms, high-order modes involve rapid induced-charge oscillations that produce sign cancellations in the interaction between distant charges, so that the effective interaction is local and each point on the particle surface primarily feels its immediate quasi-planar environment. In gold at visible fre- quencies, $\operatorname{Re}\{\varepsilon\}$ is quite steep (see Fig. 2), so that the bulk volume plasmon ( $\varepsilon=0$, that is, $\hbar \omega=3 \mathrm{eV}$ ) lies very close to the surface plasmon.

Analytical results are also available for more complex shapes in the non-retarded limit (basically those related to coordinate systems in which Poisson's equation is separable). In particular, the dipolar polarizability of a coated sphere is given by

$\alpha=3 V \varepsilon_{\mathrm{m}}$

$$
\times \frac{\left(R_{\mathrm{o}} / R_{\mathrm{i}}\right)^{3}\left(2 \varepsilon_{\mathrm{coat}}+\varepsilon\right)\left(\varepsilon_{\mathrm{coat}}-\varepsilon_{\mathrm{m}}\right)-\left(\varepsilon_{\mathrm{coat}}-\varepsilon\right)\left(2 \varepsilon_{\mathrm{coat}}+\varepsilon_{\mathrm{m}}\right)}{\left(R_{\mathrm{o}} / R_{\mathrm{i}}\right)^{3}\left(2 \varepsilon_{\mathrm{coat}}+\varepsilon\right)\left(\varepsilon_{\mathrm{coat}}+2 \varepsilon_{\mathrm{m}}\right)-2\left(\varepsilon_{\mathrm{coat}}-\varepsilon\right)\left(\varepsilon_{\mathrm{coat}}-\varepsilon_{\mathrm{m}}\right),}
$$

where $R_{\mathrm{i}}$ is the internal radius of the core material described by $\varepsilon$, the coating of permittivity $\varepsilon_{\text {coat }}$ extends up to a radius $R_{\mathrm{o}}$, and the medium outside the particle has permittivity $\varepsilon_{\mathrm{m}}$.

The polarizability of a small ellipsoid admits an analytical expression as well. The dipolar polarizability along one of the principal axes $j$ takes the form ${ }^{4}$

$$
\alpha_{j}=V \varepsilon_{\mathrm{m}} \frac{\varepsilon-\varepsilon_{\mathrm{m}}}{\varepsilon_{\mathrm{m}}+L_{j}\left(\varepsilon-\varepsilon_{\mathrm{m}}\right)}
$$

( $j=1,2,3$ denotes the principal axes of the ellipsoid), where $L_{j}$ are depolarization factors given by

$$
L_{j}=\frac{R_{1} R_{2} R_{3}}{2} \int_{0}^{\infty} \frac{\mathrm{d} s}{\left(s+R_{j}^{2}\right) \sqrt{\left(s+R_{1}^{2}\right)\left(s+R_{2}^{2}\right)\left(s+R_{3}^{2}\right)}}
$$

and satisfying the condition $L_{1}+L_{2}+L_{3}=1$, and $R_{j}$ are the corresponding lengths of the half-axes of the ellipsoid. The sphere is recovered for $R_{1}=R_{2}=R_{3}$, which yields $L_{j}=1 / 3$. For ellipsoids of revolution, with $R_{1}=R_{2}$, the depolarization factors depend on the aspect ratio $R_{3} / R_{1}$ as shown in Fig. 3. The plasmon resonances are given by the zeros of the denominator in eqn (4), $\varepsilon=\left(-1 / L_{j}+1\right) \varepsilon_{\mathrm{m}}$, implying intense light scattering. As the particle becomes more elongated along the $j=3$ axis, $L_{3}$ decreases (see Fig. 3) and the longitudinal resonance requires larger values of $-\operatorname{Re}\{\varepsilon\}$, which occur at longer wavelengths (i.e., more to the red) according to Fig. 2.

Finally, it is useful to note that dipolar contributions to the far-field cross sections can be obtained from the polarizability

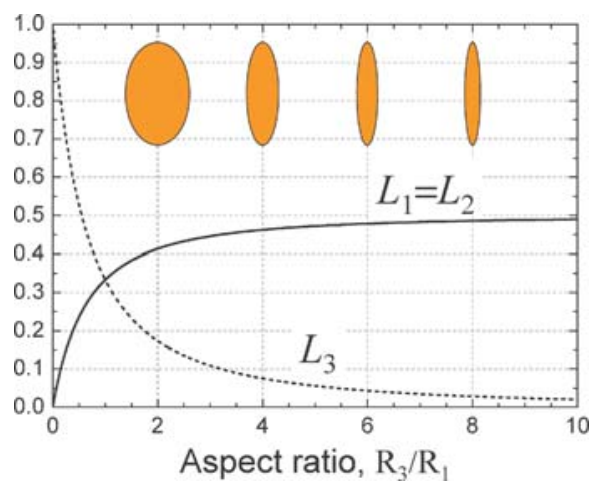

Fig. 3 Depolarization factors for axially-symmetric ellipsoids as a function of their aspect ratio. 
$\alpha$ using the following expressions:

$$
\begin{gathered}
\sigma_{\mathrm{abs}}+\sigma_{\mathrm{sc}}=\frac{2 \pi}{\lambda \sqrt{\varepsilon_{\mathrm{m}}}} \operatorname{Im}\{\alpha\}, \\
\sigma_{\mathrm{sc}}=\frac{8 \pi^{3}}{3 \lambda^{4}}|\alpha|^{2} .
\end{gathered}
$$

\section{Retardation effects. Mie theory}

The finiteness of the speed of light has important consequences that affect the response of gold nanoparticles. First of all, the electromagnetic field cannot penetrate beyond a certain depth inside the metal, the so-called skin depth, which is of the order of $15 \mathrm{~nm}$ in the vis-NIR. But more importantly, redshifts take place as the particle size increases, and retardation effects play a significant role when the diameter $2 R$ is a sizeable fraction of the mode wavelength $\lambda_{\mathrm{m}}$ in the surrounding medium, which is related to the free-space wavelength through $\lambda_{\mathrm{m}}=\lambda / \sqrt{\varepsilon_{\mathrm{m}}}$ (the effect is clearly visible even for a radius as small as $R \approx 0.1 \lambda_{\mathrm{m}}$,
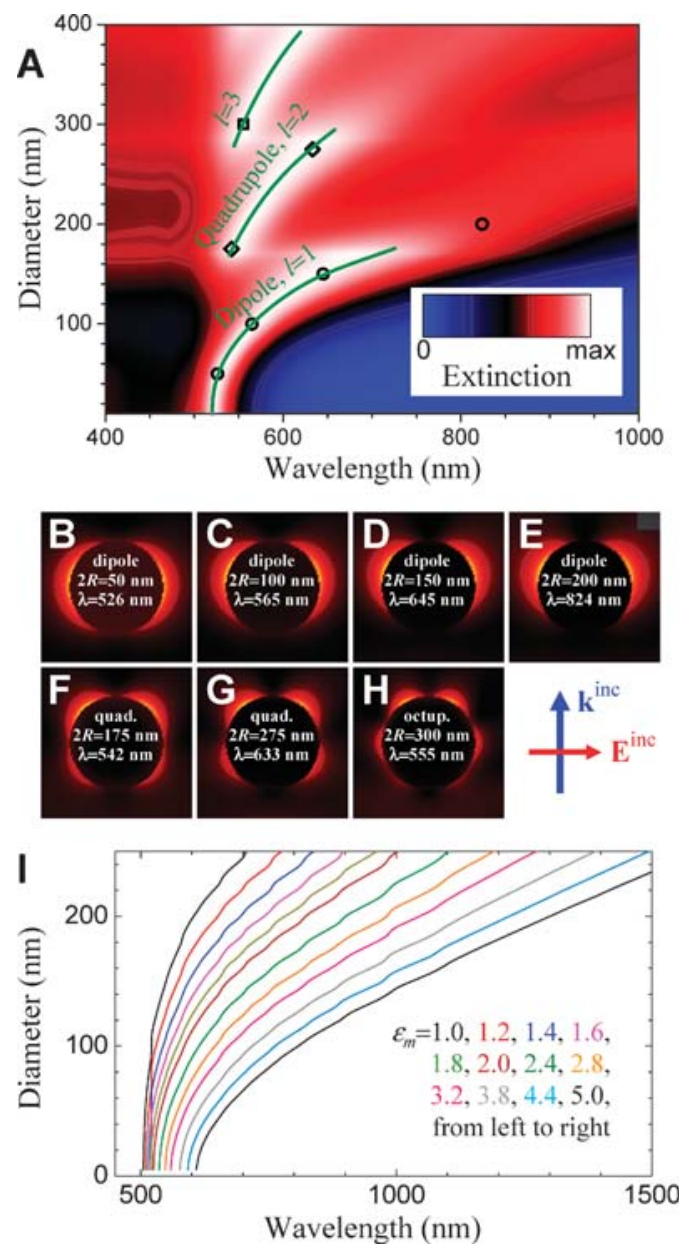

Fig. 4 (A) Evolution of extinction spectra over a wide range of particle sizes for gold nanospheres in water, normalized to the maximum extinction for each value of the diameter, $2 R$. (B-H) Near-field enhancement maps for dipole, quadrupole, and $l=3$ modes in particles of different diameters, corresponding to the symbols superimposed in the upper contour plot. (I) Dipole mode for gold spheres in different dielectric environments. as shown in Fig. 4). In particular, opposite charges are separated by roughly one particle diameter in a dipole mode, so that the reaction of one end of the particle to changes produced in the other end takes place with a phase delay of the order of $4 \pi R / \lambda_{\mathrm{m}}$, and consequently, the period of one mode oscillation increases to accommodate this delay. Quadrupoles and higher-order modes produce more nodes in the distribution of the polarization charges induced on the surface of the particle (the number of nodes is proportional to $l$ ), which reduce the effective interaction distance, so that the shift of an $l$ mode in a particle of radius $R$ is comparable to the dipole shift for radius $R / l$ (see Fig. 4(A)).

These effects are clearly observed in spherical gold particles, for which the analytical solution of Maxwell's equations was first found by Mie. ${ }^{3}$ The total cross section of a spherical particle becomes

$$
\sigma=\frac{\lambda_{\mathrm{m}}^{2}}{2 \pi} \sum_{l=1}^{l=\infty}(2 l+1)\left[\operatorname{Im}\left\{t_{l}^{\mathrm{E}}\right\}+\operatorname{Im}\left\{t_{l}^{\mathrm{M}}\right\}\right],
$$

where $t_{l}^{\mathrm{E}}$ and $t_{l}^{\mathrm{M}}$ are electric and magnetic scattering coefficients, respectively. These coefficients admit analytical expressions in terms of spherical Bessel and Hankel functions for a homogeneous sphere: ${ }^{19}$

$$
\begin{gathered}
t_{l}^{\mathrm{E}}=\frac{-\varepsilon_{\mathrm{m}} j_{l}\left(\rho_{\mathrm{m}}\right)\left[j_{l}(\rho)+\rho j_{l}^{\prime}(\rho)\right]+\varepsilon\left[j_{l}\left(\rho_{\mathrm{m}}\right)+\rho_{\mathrm{m}} j_{l}^{\prime}\left(\rho_{\mathrm{m}}\right)\right] j_{l}(\rho)}{\varepsilon_{\mathrm{m}} h_{l}^{(+)}\left(\rho_{\mathrm{m}}\right)\left[j_{l}(\rho)+\rho j_{l}^{\prime}(\rho)\right]-\varepsilon\left[h_{l}^{(+)}\left(\rho_{\mathrm{m}}\right)+\rho_{\mathrm{m}} h_{l}^{(+)^{\prime}}\left(\rho_{\mathrm{m}}\right)\right] j_{l}(\rho)}, \\
t_{l}^{\mathrm{M}}=\frac{-\rho j_{l}\left(\rho_{\mathrm{m}}\right) j_{l}^{\prime}(\rho)+\rho_{\mathrm{m}} j_{l}^{\prime}\left(\rho_{\mathrm{m}}\right) j_{l}(\rho)}{\rho h_{l}^{(+)}\left(\rho_{\mathrm{m}}\right) j_{l}^{\prime}(\rho)-\rho_{\mathrm{m}} h_{l}^{(+)^{\prime}}\left(\rho_{\mathrm{m}}\right) j_{l}(\rho)},
\end{gathered}
$$

where $\rho=(2 \pi R / \lambda) \sqrt{\varepsilon}, \rho_{\mathrm{m}}=(2 \pi R / \lambda) \sqrt{\varepsilon_{\mathrm{m}}}$, and the primes stand for derivatives. Plasmon modes are related to the poles of $t_{l}^{\mathrm{E}}$, whereas the magnetic contribution to scattering can only produce (broad) resonances for particle sizes comparable to the wavelength. The multipolar polarizability is proportional to $t_{l}^{\mathrm{E}}$, and in particular, the dipolar polarizability reads $\alpha=\left(3 \lambda^{3} / 4 \pi^{2}\right) t_{l=1}^{\mathrm{E}}$, where the dipolar electric scattering coefficient is readily calculated using $j_{1}(x)=\sin x / x^{2}-\cos x / x$ and $h_{1}^{(+)}(x)=\left(1 / x^{2}-i / x\right) \exp (i x)$. This value of $\alpha$ is actually very accurate for describing gold spheres with diameters up to $\sim 150 \mathrm{~nm}$. The following simplified expression for $\alpha$ is obtained for small radii: ${ }^{30}$

$$
\alpha=3 V \varepsilon_{\mathrm{m}} \frac{1-0.1\left(\varepsilon+\varepsilon_{\mathrm{m}}\right) \theta^{2} / 4}{\frac{\varepsilon+2 \varepsilon_{\mathrm{m}}}{\varepsilon-\varepsilon_{\mathrm{m}}}-\left(0.1 \varepsilon+\varepsilon_{\mathrm{m}}\right) \theta^{2} / 4-i(2 / 3) \varepsilon_{\mathrm{m}}^{3 / 2} \theta^{3}},
$$

where $\theta=2 \pi R / \lambda$ is the size parameter. The electrostatic limit of eqn (3) is recovered for $\theta=0$. The second and third terms in the denominator are corrections contributing mainly to the dipolar plasmon shift and broadening, respectively. ${ }^{30}$

The exact cross section of gold spheres in water calculated from eqn (5) is represented in Fig. 4(A) as a function of diameter and wavelength, showing red-shifts with increasing particle size and higher-order multipoles emerging in large particles. The near-field plots corresponding to these modes (Fig. 4(B-H)) show two, four, and six bright regions for dipoles, quadrupoles, and $l=3$ modes, respectively, ${ }^{3}$ although multipole mixing leads to a modulation in the actual position 


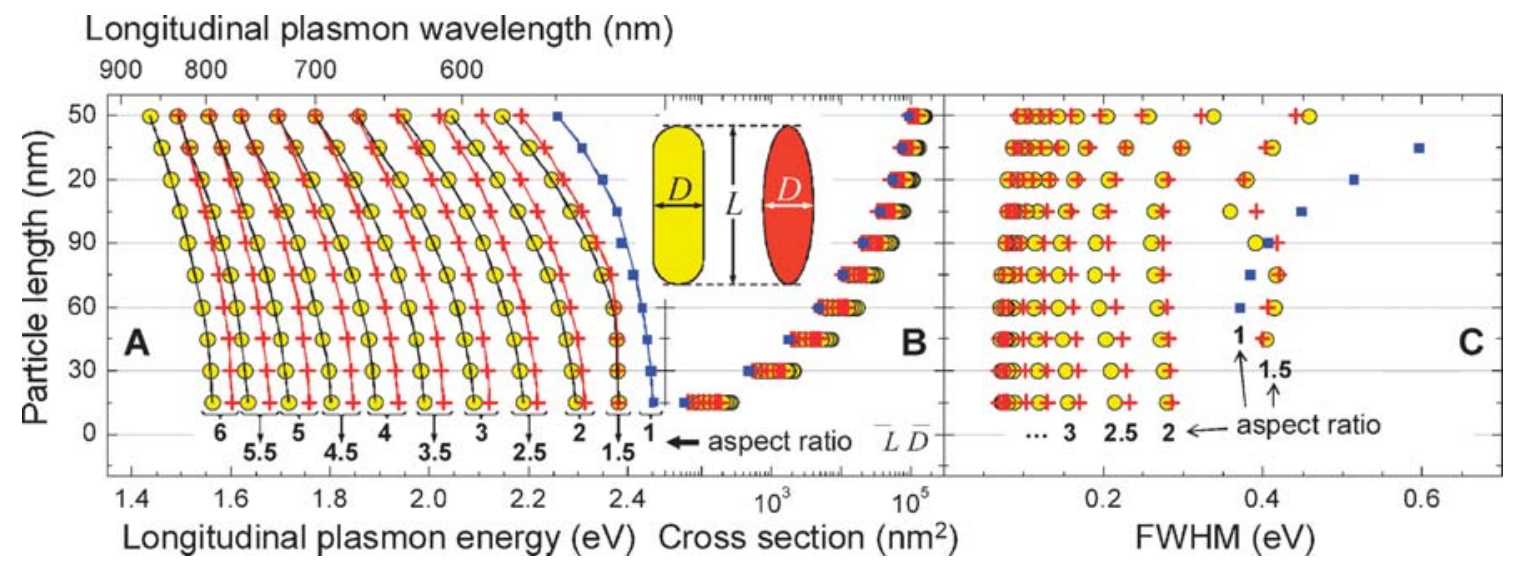

Fig. 5 (A) Longitudinal plasmon resonance wavelength in gold ellipsoids (red + symbols) and rods with hemispherical caps (yellow circles) as a function of particle length (vertical axis) and aspect ratio (labels at the bottom). Dipolar plasmons in spheres (unit aspect ratio) are shown as a reference (blue squares). All data are for particles in air. (B) Extinction cross section at peak maximum for illumination with incident electric field along the particle's axis of symmetry. The extinction increases with aspect ratio for fixed length. (C) Full width at half maximum of the longitudinal plasmon mode. The width decreases with increasing aspect ratio.

and magnitude of the field intensity maxima. It should be emphasized that the particle responds basically as an induced dipole for diameters below $\sim 150 \mathrm{~nm}$. However, higher-multipole modes become dominant at larger sizes. These multipoles are also relevant in the interaction between neighbouring particles, even for small diameters (see section 9).

A similar increase in the importance of retardation effects with particle size is observed for other shapes, such as gold rods and ellipsoids, as is summarised in Fig. 5, where the wavelength, full-width at half maximum (FWHM), and maximum cross section of the main longitudinal plasmon mode are plotted as a function of particle size and aspect ratio. These results were obtained using BEM (see section 5.1). The longitudinal mode has a dipolar character, with a $(+-)$ charge pattern along the major axis, and is excited by light with incident field along that axis. An approximate expression for the polarizability of gold ellipsoids that follows the form of eqn (6) has been proposed, ${ }^{30}$ based upon fitting constants obtained from data similar to those of Fig. 5, with excellent results compared to both experiment and rigorous calculations.

A red shift is observed with increasing aspect ratio and particle length in both rods and ellipsoids. The red-shift seen when increasing the size has a similar retardation origin as that for spheres. The red-shift for higher aspect ratios with fixed length is produced by shape-dependent depolarization, as shown in Fig. 3 for ellipsoids in the non-retarded limit. Interestingly, the plasmon resonance becomes narrower with increasing aspect ratio (see Fig. 5(C)) as a result of lower absorption accompanying the red shift of the mode (the absorption is roughly controlled by the ratio $\operatorname{Im}\{\varepsilon\} /|\varepsilon|$, which drops with increasing wavelength). In contrast, radiative losses increase noticeably in longer particles, where they contribute to make the plasmon resonance broader.

The sensitivity to the dielectric environment is a central feature of nanoparticle optics and determines the capability to sense the presence of attached macromolecules. The plasmon modes shift towards larger values of $-\operatorname{Re}\{\varepsilon\}$ as the permittivity of the surrounding medium $\varepsilon_{\mathrm{m}}$ increases (see eqn (3)), which translates into a red-shift according to Fig. 2. This is shown in
Fig. 4(I) for a wide range of $\varepsilon_{\mathrm{m}}$ values. Furthermore, the red-shift due to retardation becomes significant at smaller particle sizes due to the decrease in $\lambda_{\mathrm{m}}=\lambda / \sqrt{\varepsilon_{\mathrm{m}}}$.

\section{Non-local effects}

We have so far explained a story that works well for relatively big particles, but a description of gold through its local bulk permittivity $\varepsilon(\omega)$ fails dramatically for small diameters, below $\sim 10 \mathrm{~nm}$, that is, when the particle size is comparable to the mean free path of conduction electrons. These so-called finitesize effects have been phenomenologically accounted for by increasing the damping rate of the conduction electrons contribution to the permittivity, ${ }^{31}$ so that the particle is described through the modified dielectric function

$$
\varepsilon(\omega)+\frac{\omega_{\mathrm{p}}^{2}}{\omega\left(\omega+i \tau^{-1}\right)}-\frac{\omega_{\mathrm{p}}^{2}}{\omega\left(\omega+i \tau^{-1}+i v_{\mathrm{F}} / R\right)},
$$

where $v_{\mathrm{F}}$ is the Fermi velocity $\left(1.39 \times 10^{8} \mathrm{~cm} \mathrm{~s}^{-1}\right.$ in gold $)$ and $R$ is the particle radius. In other words, the damping rate $\tau^{-1}$ in the conduction electron motion is increased by the rate of collisions with the particle surface for electrons moving near

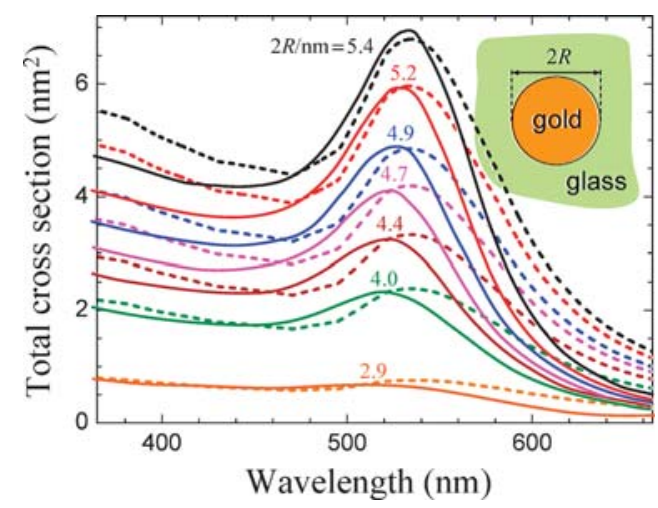

Fig. 6 Light scattering and absorption by small gold particles. Experiment ${ }^{31}$ (solid curves) is compared to finite-size-corrected local theory (broken curves) based upon the permittivity of eqn (7). 
the Fermi level (i.e., those actually determining the collective plasmon response). This procedure brings theory into reasonable agreement with experiment, as shown in Fig. 6, and it is quite different from the nearly size-independent spectral profile predicted without finite-size corrections for small $R$. It should be noted however that the recipe just outlined is not so well defined for non-spherical particles (or even for shells, in which the distance travelled by conduction electrons before they hit a metal boundary is not solely determined by the radius, but also by the shell thickness). Furthermore, non-local effects are expected to play a significant role in sharp corners of bigger particles, but no methods are currently available to successfully describe these situations.

\section{Numerical methods}

The complexity of the electromagnetic field in the presence of arbitrarily-shaped nanoparticles is such that Maxwell's equations must be solved using numerical methods. Here we describe the most popular and efficient approaches, and provide a critical comparison of their capabilities for practical cases. The local approximation is common to all these methods (i.e., the different materials are described by dielectric functions that depend only on the frequency of light).

\subsection{Boundary element method (BEM)}

The methods described in this section and in section 5.2 produce solutions of Maxwell's equations in frequency space $\omega$, and consequently all the electromagnetic quantities are assumed to have an $\exp (-i \omega t)$ time dependence, and, for example, the solution for the electric field $\boldsymbol{E}(\boldsymbol{r})$ obtained from these methods permits the time-dependent electric field to be written as $\boldsymbol{E}(\boldsymbol{r}, t)=\boldsymbol{E}(\boldsymbol{r}) \exp (-i \omega t)+\boldsymbol{E}^{*}(\boldsymbol{r}) \exp (i \omega t)$.

The electromagnetic field inside each homogeneous region in a composite material is unambiguously determined by the fields and their derivatives at the boundary of that region, or equivalently, by a distribution of charges and currents on that boundary. This is a rigorous result derived from vector diffraction theory ${ }^{27}$ that is the basis of BEM. This method starts by expressing the electromagnetic field scattered by a nanoparticle in terms of boundary charges and currents, which, upon imposing the customary boundary conditions for the continuity of the parallel components of the electric and magnetic fields, leads to a system of surface-integral equations. This system is solved by discretizing the integrals using a set of $N$ representative points distributed at the boundaries, so that it turns into a set of linear equations that are solved numerically by standard linear-algebra techniques. ${ }^{15}$

More specifically, the electric field is first expressed in terms of scalar and vector potentials $\phi$ and $\boldsymbol{A}$ as

$$
\boldsymbol{E}(\boldsymbol{r})=i k \boldsymbol{A}(\boldsymbol{r})-\nabla \phi(\boldsymbol{r}),
$$

and these potentials are in turn rigorously written as

$$
\phi(\boldsymbol{r})=\phi_{j}^{\mathrm{e}}(\boldsymbol{r})+\int_{S_{j}} \mathrm{~d} s G_{j}(\boldsymbol{r}-\boldsymbol{s}) \sigma_{j}(\boldsymbol{s})
$$

and

$$
\boldsymbol{A}(\boldsymbol{r})=\boldsymbol{A}_{j}^{\mathrm{e}}(\boldsymbol{r})+\int_{S_{j}} \mathrm{~d} s G_{j}(\boldsymbol{r}-\boldsymbol{s}) \boldsymbol{h}_{j}(\boldsymbol{s}),
$$

valid for $\mathbf{r}$ inside medium $j$, where the integrals are extended over the boundary of each medium $j$, the function

$$
G_{j}(\boldsymbol{r}-\boldsymbol{s})=\frac{\mathrm{e}^{i k_{j}|\boldsymbol{r}-\boldsymbol{s}|}}{4 \pi|\boldsymbol{r}-\boldsymbol{s}|}
$$

takes care of the propagation of each scalar component of the fields inside medium $j$ (e.g., in a colloidal dispersion we can take $j=1$ for gold and $j=2$ for the solvent); $k_{j}=\sqrt{\varepsilon_{j}} \omega / c$ is the light wavevector inside that medium, characterised by the dielectric function $\varepsilon_{j} ; \sigma_{j}$ and $\boldsymbol{h}_{j}$ are the abovementioned boundary charge and current distributions, respectively; $\phi_{j}^{\mathrm{e}}$ and $\boldsymbol{A}_{j}^{\mathrm{e}}$ are the scalar and vector potentials of the incident light; and the integral is extended over the boundary of medium $j$. In particular, for external plane-wave illumination, one can take $\phi_{j}^{\mathrm{e}}=0$, while the external vector potential is equal to a plane wave in the solvent and 0 inside the particle.

One of the boundary conditions is the continuity of the scalar potential across the interface between two media, leading to the condition

$$
\begin{aligned}
& \phi_{1}^{\mathrm{e}}(\boldsymbol{s})+\int_{S_{1}} \mathrm{~d} \boldsymbol{s}^{\prime} G_{1}\left(\boldsymbol{s}-\boldsymbol{s}^{\prime}\right) \sigma_{1}\left(\boldsymbol{s}^{\prime}\right) \\
& \quad=\phi_{2}^{\mathrm{e}}(\boldsymbol{s})+\int_{S_{2}} \mathrm{~d} \boldsymbol{s}^{\prime} G_{2}\left(\boldsymbol{s}-\boldsymbol{s}^{\prime}\right) \sigma_{2}\left(\boldsymbol{s}^{\prime}\right) .
\end{aligned}
$$

It is convenient to use matrix notation and write this expression as

$$
G_{1} \sigma_{1}-G_{2} \sigma_{2}=\phi_{2}^{\mathrm{e}}-\phi_{1}^{\mathrm{e}}
$$

This notation is particularly suitable to deal with the discretized system, in which each surface integral is approximated by the sum

$$
\int_{S_{j}} \mathrm{~d} \boldsymbol{s}^{\prime} G_{j}\left(\boldsymbol{s}_{l}-\boldsymbol{s}^{\prime}\right) \sigma_{j}\left(\boldsymbol{s}^{\prime}\right)=\sum_{l^{\prime}} G_{j, l l^{\prime}} \sigma_{j}\left(\boldsymbol{s}_{l^{\prime}}\right) \Delta S_{j, l^{\prime}},
$$

with $l^{\prime}$ running over a finite set of $N$ discretization points $\boldsymbol{s}_{l^{\prime}}$ (these are taken at the centre of their respective surface elements $\Delta S_{j, l}$, which define a complete tessellation of the boundary $S_{j}$ ). The matrix elements $G_{j, l l^{\prime}}$ are defined as

$$
G_{j, l l^{\prime}}=\int_{\Delta S_{j, l^{\prime}}} \mathrm{d} \boldsymbol{s}^{\prime} G_{j}\left(\boldsymbol{s}_{l}-\boldsymbol{s}^{\prime}\right)
$$

assuming that the surface charge varies smoothly over the interface and does not change significantly within the extension of each surface element $\Delta S_{j, l}$, so that it can be pulled outside the integral. Similar equations are obtained for the remaining boundary conditions, involving both interface charges and currents. More details are given in ref. 15 .

Taking advantage of particle symmetry can be very advantageous for the calculation. For instance, for particles possessing axial symmetry, we can expand the fields in terms of azimuthal components with $\exp (\operatorname{im} \varphi)$ dependence on the azimuthal angle $\varphi$. Then, the BEM equations can be solved independently for each azimuthal component $m$, and the $\varphi$ part of the surface integrals can be carried out before discretizing the system. Only the contour of the particle needs to 


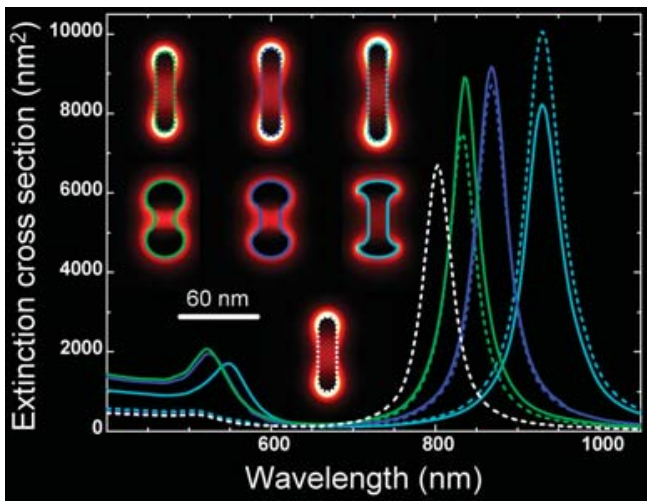

Fig. 7 Extinction spectra of axially-symmetric gold dumbbells of total length $L=60 \mathrm{~nm}$ and aspect ratio 4 for increasing diameter of the thick ends (solid curves) compared to straight rods with hemispherical caps and the same central diameter (broken curves). All spectra are for particles in water. The lengths of the straight rods (64 $\mathrm{nm}, 69 \mathrm{~nm}$, and $76 \mathrm{~nm}$, corresponding to aspect ratios 4.3, 4.6, and 5.1, respectively) have been adjusted to yield the same longitudinal plasmon wavelength as the dumbbells. The straight rod of length $L=60$ $\mathrm{nm}$ is also shown for comparison. The central diameter is $15 \mathrm{~nm}$ in all particles. The particle profiles and near-field distributions of the longitudinal plasmon mode are shown in the insets. The spectra are averaged over particle orientations and incident light polarizations.

be parametrized in that case, so that the dimensions of the system effectively reduce to one (the contour) rather than two (the surface boundary), and therefore $N$ becomes of the order of hundreds rather than thousands of points.

The axial version of $\mathrm{BEM}^{15}$ has actually been used to calculate the data shown in Fig. 5. Another example of the use of BEM is offered in Fig. 7 for dumbbells compared to rods, demonstrating that the effective aspect ratio of dumbbells increases when flattening the ends. In fact, flatter ends can gather more induced charge (this is the origin of the larger field enhancement in this type of geometry) during each optical cycle of the longitudinal dipole mode (at wavelengths of 800-1000 nm), which take a longer time to move from one end of the particle to the other for a fixed value of the central diameter. This flattening of the tips also results in red shifts and enhancement of the lower-wavelength transversal modes (below $600 \mathrm{~nm}$ ), since they can be regarded as disks of increasing aspect ratio, with weak coupling to the central rod for transverse polarization. Fully-converged spectra of this sort involve 100-200 points per particle and require just a few minutes on a workstation.

The solution of the BEM equations for less symmetric particles, such as those considered in Fig. 8, takes a few hours per spectrum and involves $\sim 5000$ parametrization points. This figure shows snapshots of the evolution from circular rods to octahedra during colloidal synthesis. ${ }^{16,32}$ Agreement between experiment (solid curves) and theory (dashed curves) is quite satisfactory. The evolution from a single dominant longitudinal plasmon mode into the main plasmon of the symmetric octahedron, passing through the two plasmon modes of the intermediate low-aspect-ratio rods with square cross sections, is well described by the theory, and so is the colour of particle dispersions either in transmission (background colour in the plots) or in reflection (colours on the particle surfaces).

Further examples of BEM calculations are given in Fig. 9 for gold decahedra, which are discussed in section 5.2 and compared with calculations using the discrete-dipole approximation.

\subsection{Discrete-dipole approximation (DDA)}

The discrete dipole approximation (DDA), also called the coupled-dipole method (CDM), derives its name from the description of the metal as an array of polarizable dipolar elements organised on a lattice to represent the shape of the nanoparticle. Like BEM, this method yields solutions of the electromagnetic field in response to an incident electric field $\boldsymbol{E}^{\mathrm{inc}}(\boldsymbol{r})$ in the frequency domain. The polarization of each

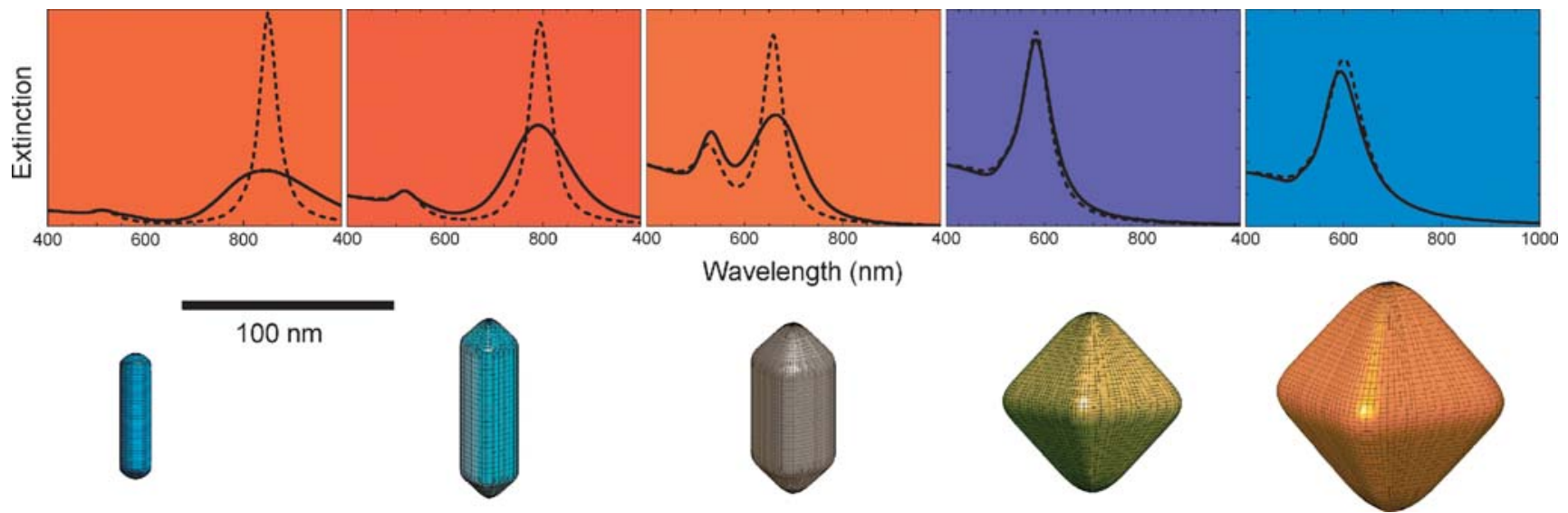

Fig. 8 The colours of gold nanoparticles. Under suitable conditions, ${ }^{32}$ small circular gold rods (left, length $L=60 \mathrm{~nm}$ and diameter $a=15.5 \mathrm{~nm}$ ) can be made to grow into large octahedra (right, height $L=109 \mathrm{~nm}$ ) through intermediate stages involving square rods of decreasing aspect ratio (length $L=85 \mathrm{~nm}$ and side $a=23 \mathrm{~nm}$ in the second frame from the left, followed by $L=81 \mathrm{~nm}$ and $a=34$ nm in the centre) and small octahedra $(L=85 \mathrm{~nm})$. Measured spectra at intermediate stages of this reaction ${ }^{32}$ (solid curves) are modelled here by applying BEM to shapes as shown in the lower insets, ${ }^{16}$ with dimensions and rounding of both edges and corners as obtained from transmission electron microscope (TEM) images of the particles. The particles are surrounded by DMF $\left(\varepsilon_{\mathrm{m}}=2.04\right)$ and they are painted here in the colours of the light that they scatter when illuminated with a white source. The background colour in each plot corresponds to the light transmitted through a dilute colloidal dispersion. 

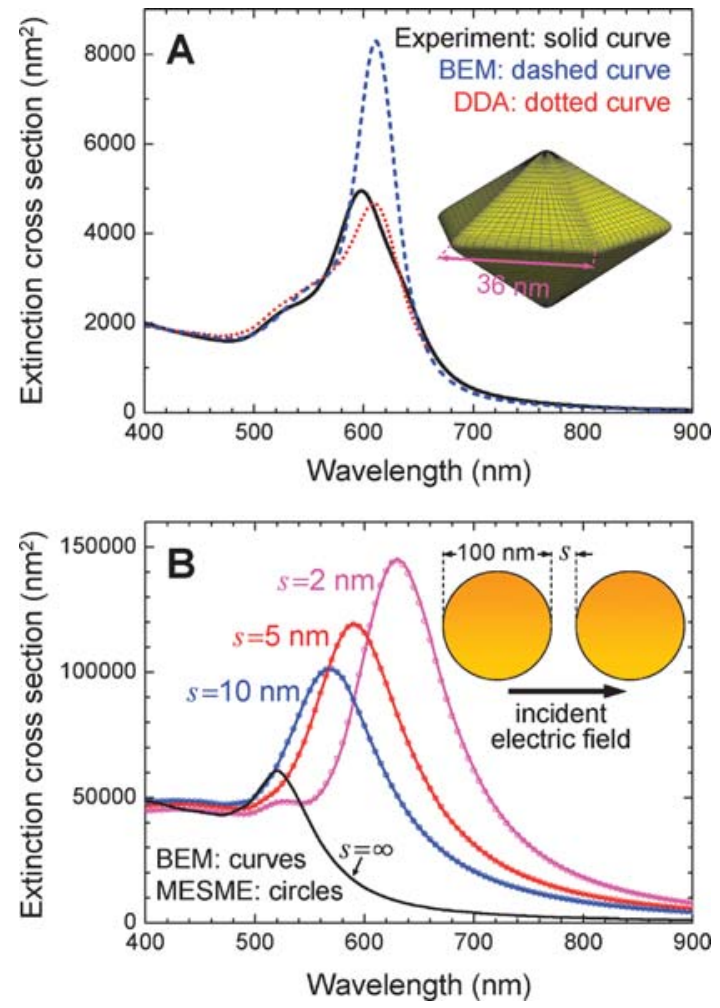

Fig. 9 (A) Extinction of small gold decahedra in DMF calculated with BEM (dashed curve) and DDA (dotted curve), compared to experiment (solid curve). All curves are normalized to the BEM result at a wavelength of $400 \mathrm{~nm}$. (B) Extinction of gold dimers for longitudinal field illumination and different distances between sphere surfaces, calculated with BEM (solid curves) and MESME (circles).

element is a result of the interaction with the local electromagnetic field produced by all other elements plus the external field. In particular, the dipole in the element occupying the position $\boldsymbol{r}_{j}$ satisfies the self-consistent relation ${ }^{6,7}$

$$
\boldsymbol{p}_{j}=\alpha_{j}\left[\boldsymbol{E}^{\mathrm{inc}}\left(\boldsymbol{r}_{j}\right)+\sum_{j^{\prime} \neq j} \boldsymbol{E}_{j}^{\mathrm{scat}}\left(\boldsymbol{r}_{j}\right)\right],
$$

where

$$
\boldsymbol{E}_{j^{\prime}}^{\mathrm{scat}}\left(\boldsymbol{r}_{j}\right)=\frac{e^{i k_{\mathrm{m}} R_{j j^{\prime}}}}{4 \pi \varepsilon_{\mathrm{m}} R_{j j^{\prime}}^{3}}\left[A_{j j^{\prime}} \boldsymbol{p}_{j^{\prime}}-B_{j j^{\prime}} \frac{\boldsymbol{R}_{j j^{\prime}}\left(\boldsymbol{R}_{j j^{\prime}} \cdot \boldsymbol{p}_{j^{\prime}}\right)}{R_{j j^{\prime}}^{2}}\right]
$$

is the scattered field produced by element $j^{\prime}$ at the position of element $j, k_{\mathrm{m}}=2 \pi / \lambda_{\mathrm{m}}$ is the wavevector of light in the surrounding medium of permittivity $\varepsilon_{\mathrm{m}}$, and we have defined the scalar coefficients $A_{j j^{\prime}}=\left(k_{\mathrm{m}} R_{j j^{\prime}}\right)^{2}+i k_{\mathrm{m}} R_{j j^{\prime}}-1$ and $B_{j j^{\prime}}=$ $\left(k_{\mathrm{m}} R_{i j j^{\prime}}\right)^{2}+3 i k_{\mathrm{m}} R_{i j^{\prime}}-3$, and also the coordinate difference $\boldsymbol{R}_{j j^{\prime}}=\boldsymbol{r}_{j}-\boldsymbol{r}_{j^{\prime}}$. These equations can be extended to particles on a substrate, such as those produced lithographically, by including the image dipoles as well as the reflection of $\boldsymbol{E}^{\text {inc }}(\boldsymbol{r})$. The polarizability $\alpha_{j}$ of element $j$ can be obtained from the Clausius-Mossotti relation (see section 10.1) using as input the volume of the element and the permittivity of the medium in which it is sitting ${ }^{6}$ (i.e., DDA will trivially yield the bulk dielectric function in an infinite homogeneous medium), although more sophisticated and accurate prescriptions have been formulated for $\alpha_{j}$, including radiative-reaction corrections. ${ }^{33}$ A considerable improvement in computational efficiency is achieved by solving the DDA eqn (8) iteratively using the conjugate gradient method (CGM) and performing matrix-vector products in reciprocal space through the fastFourier transform (FFT), ${ }^{34}$ as shown in Table 1 below, particularly considering that $>10^{4}$ elements are typically required in practice.

The freely-available DDSCAT implementation ${ }^{8}$ of the DDA method incorporates all of the above features. There have been numerous applications of this method to model absorption and scattering spectra of particles with different shapes, including plasmonic bands. ${ }^{9,35}$

There are several related methods that use volume partitions, like the method of moments, volume integral formulations, and the Green function approach. ${ }^{36}$ All of these are reformulations of the electromagnetic field in terms of volume elements, and are exact in the limit of small elements. In fact, the DDA eqn (8) can be obtained from discretization of integral equations for the electric field.

Fig. 9(A) presents a comparison of BEM (dashed curve) and DDA (dotted curve) calculations with the corresponding ensemble experimental spectrum ${ }^{37}$ for gold decahedra in DMF. The converged BEM calculations involved 6000 points and required 15 minutes per wavelength. DDA calculations depend critically on the discretization step, which needs to be small compared to the skin depth. Both calculations yield a plasmon peak relatively close to the experimental value (solid curve). The latter is broadened due to finite size and shape distribution of the nanoparticle ensemble, which explains why BEM yields a narrower width for a fixed particle shape and size.

\subsection{Other approaches}

In contrast to the two approaches discussed above, the finite difference in the time domain method (FDTD) relies on propagation of the electromagnetic field defined on a spatial grid through consecutive time steps, so the method yields $\boldsymbol{E}(\boldsymbol{r}, t)$ in a direct way. ${ }^{13,38}$ This can be advantageous in situations for which time-resolved fields are required. Furthermore, FDTD is routinely employed to simulate absorption and scattering spectra by time propagation of an incident field that is switched on at time $t=0$, so that the weight of each frequency component is simply given by the integral $\int_{0}^{\infty} \mathrm{d} t$ $\exp \left[i\left(\omega+i 0^{+}\right) t\right]=i / \omega$. Taking this into account, spectral information is gathered by applying FFT to the time-dependent fields. Entire spectra are obtained from a single run in time evolution and the spectral resolution is dictated by the timepropagation window through $\Delta \omega \approx 1 / t_{\max }$ (i.e., the Fourier uncertainty principle), whereas the maximum accessible frequency depends on the time step as $\omega_{\max } \ll \Delta t$. Clever procedures have been devised to incorporate artificial absorbing boundary conditions (ABCs) in order to avoid spurious reflections from the borders of the finite discretized volume in which the time propagation is carried out. ${ }^{13}$ Likewise, extensions of the method to describe dispersive materials with Drude-like dielectric functions are now well developed. In particular, FDTD can be used to analyze the optical properties of complex nanoparticles, such as gold nanostars. ${ }^{14}$ 
Table 1 Critical comparison of the performance of BEM, DDA, and FDTD. The computational demand in BEM and DDA is presented in terms of the number of calculated frequencies $N_{\omega}$ and the particle volume $V$, measured in units of the cube of the skin depth (this is $\sim 15 \mathrm{~nm}$ in the visNIR, and several subdivisions are needed inside the metal to describe the decay of the field within this distance). The discretized volume in FDTD $\left(V^{\prime}\right)$ extends considerably outside the particle in order to account for light propagation in the surrounding medium. Direct inversion of the secular linear equations in BEM and DDA is assumed (see footnotes for iterative solutions). The factor $\omega / \Delta \omega$ in FDTD is proportional to the number of time steps needed to describe a component of frequency $\omega$ with frequency resolution $\Delta \omega$

\begin{tabular}{|c|c|c|c|}
\hline & BEM & DDA & FDTD \\
\hline $\begin{array}{l}\text { Computational } \\
\text { demand }\end{array}$ & $V^{2} N_{\omega}^{a}$ & $V^{3} N_{\omega}^{b}$ & $V^{\prime} \omega / \Delta \omega$ \\
\hline Storage demand & $V^{4 / 3}$ & $V^{2}$ & $V^{\prime}$ \\
\hline \multirow[t]{2}{*}{ Advantages } & $\begin{array}{l}\text { - Low computational and storage } \\
\text { demand }\end{array}$ & - Simple parametrization & - Simple parametrization \\
\hline & $\begin{array}{l}\text { - Only the particle surface is } \\
\text { discretized }\end{array}$ & - Only the particle volume is discretized & - Simple implementation \\
\hline Disadvantages & - Complex parametrization & $\begin{array}{l}\text { - Time consuming } \\
- \text { Particle size } a \text { and dielectric function } \varepsilon \text { limited to } \\
|a \sqrt{\varepsilon}| / \lambda<5\end{array}$ & $\begin{array}{l}\text { - Full spectrum in single run } \\
\text { - Time consuming } \\
\text { - Difficult to apply to arbitrary } \\
\varepsilon(\omega) \\
\text { - Requires parametrization of } \\
\text { volume outside the particle and } \\
\text { ABCs }\end{array}$ \\
\hline
\end{tabular}

${ }^{a}$ BEM scales as $V^{4 / 3} N_{\omega}$ in time when using iterative methods like CGM. ${ }^{b}$ DDA scales as $V \log V N_{\omega}$ in time when using CGM accompanied by FFT. The number of iterations increases with the magnitude of the permittivity in DDA, and becomes very large for gold in the NIR.

Expansions in terms of eigenmodes of symmetric components (e.g., spherical, cylindrical and plane waves) are computationally advantageous in geometries composed of sub-systems that admit analytical solutions, like spherical multi-shells, dimers, cylinders, spheres supported on planar substrates, and sphere arrays. These frequency-domain methods use expansions of the fields in terms of analytical solutions of various parts of the system, and the expansion coefficients are determined by imposing field boundary conditions.

There are numerous examples of hybrid methods in the literature, combining several of the features of the methods so far discussed: modal expansions in certain spatial direction(s) with volume or boundary discretization in the remaining ones; plane wave expansions in the frequency domain, which is particularly suitable for periodic particle arrays, ${ }^{39}$ time propagation of plane wave expansions; methods similar to BEM or DDA but with discretization based upon suitable modal functions rather than points in space. ${ }^{40}$ However, BEM, DDA, and FDTD are by and large the most versatile, efficient, and widely-used methods, and for which we therefore present a critical comparison of advantages and disadvantages in the following section.

The generalized multipole method is particularly useful to deal with several particles simultaneously. Fig. 9(B) contemplates the power of this method, in its multiple elastic scattering of multipole expansions (MESME) implementation ${ }^{41}$ as applied to a dimer for different distances between $100 \mathrm{~nm}$ gold spheres down to a separation of $2 \mathrm{~nm}$. The MESME calculations reported in the figure (circles) take only seconds on a workstation, compared to minutes for the BEM calculations (solid curves), with both methods agreeing perfectly on the scale of the figure.

\subsection{Critical comparison}

Computational speed. Maxwell's equations are generally written as a system of linear equations in methods operating in the frequency domain, such as BEM and DDA. The solution of such systems depends on whether a large array of electric field values are required, in which case direct inversion of the secular matrix might be needed, resulting in the computation time scaling shown in Table 1 (i.e., as the cube of the number of discretization points, $N^{3}$ ). Faster computational scaling is obtained however using iterative procedures (e.g., $N^{2}$ in CGM). BEM has the advantage that it requires parametrization only of the surface of the particle (i.e., $N \approx V^{2 / 3}$, where $V$ is the particle volume). In contrast, DDA relies on volume parametrization $(N \approx V)$.

FDTD requires a larger parametrization volume, which has to include a region outside the particles where both incident and reflected fields are allowed to propagate. On the positive side, the computational demand of FDTD grows only linearly with the number of parametrization points.

All three methods benefit from adaptive grids that assign finer discretization to regions where the fields are expected to vary more rapidly (e.g., inside the gold particle, and particularly near corners and edges). The computation of the BEM and DDA equations can be accelerated using iterative methods, such as CGM, particularly when combined with FFT in the case of DDA (see Table 1), although FFT prevents the use of adaptive grids.

Particle symmetry can be easily implemented in BEM and DDA (without FFT in the latter), even if the light source breaks that symmetry. Each mirror plane leads to a reduction by a factor of 4 when direct inversion of the secular equations is used, or a factor of 2 when using CGM. Similarly, each $n$ axis of rotation gives a reduction by a factor of $n^{2}$ for direct inversion and $n$ for CGM.

Storage demand. In general, memory is not an issue in the simulation of nanoparticle optical properties with currently available computers. BEM and DDA require storing matrices that connect every pair of discretization points (i.e., a memory proportional to $N^{2}$ ), whereas FDTD uses only the field at each 
time step (i.e., memory $\approx N$ ). This gives rise to the volume scaling indicated in Table 1.

Versatility. All three methods discussed in this section can be applied to arbitrarily complex geometries and frequencydependent dielectric functions, although FDTD requires the permittivity to be expressed as a sum over a limited number of Lorentzians. DDA and FDTD rely on simple volume parametrizations, which can be advantageous when very complex geometries are considered. BEM and DDA apply to separated particles without any need to parametrize the medium between them, whereas FDTD can become prohibitively time consuming in this case. BEM can be advantageous when sharp corners and narrow gaps between metal surfaces are considered (e.g., see Fig. 9(B)).

\section{Electron energy-loss spectroscopy (EELS)}

While all the examples presented above are focused on the far field, it is also important to study the near field, which provides an even richer understanding of the particle response. Scanning near-field optical microscopy (SNOM) is a widely used technique to probe the near field with typical spatial resolution of the order of $50 \mathrm{~nm}$, which is not sufficient to resolve optical details in particles of similar size. In contrast, EELS can probe metallic particles with spatial resolution below $1 \mathrm{~nm}$ and it is by far the best technique, combining high spatial and spectral resolution with near-field spectral imaging.

The EELS technique is performed within transmission electron microscopes (TEMs) equipped with electron energy analyzers. The electromagnetic perturbation produced in the particles by a passing energetic electron moving with energies in the $100-300 \mathrm{keV}$ range (i.e., with a velocity of $50-80 \%$ of the speed of light) is sampled by the electron itself, which experiences the retarding force caused by the induced field acting back on the projectile. The energy lost by the electron is released to create excitations in the particle, so that analysis of the energy loss distribution yields information on those excitations. The induced field can be decomposed into components which oscillate at different frequencies $\omega$, producing specific energy losses $\hbar \omega$, which can be resolved by an electron analyzer. The probability $\Gamma(\omega)$ that the electron undergoes such an energy loss is related to the induced electric field in the near-field region as

$$
\Gamma(\omega)=\frac{e}{\pi \hbar \omega} \int \mathrm{d} t \Re e\left\{e^{-i \omega t} \boldsymbol{v} \cdot \boldsymbol{E}^{\mathrm{ind}}\left[\boldsymbol{r}_{\mathrm{e}}(t), \omega\right]\right\},
$$

where $\boldsymbol{E}^{\mathrm{ind}}(\boldsymbol{r}, \omega)$ is the $\omega$ component of the induced field (calculated with the methods of section 5), $v$ is the electron velocity, and the integral is extended over the electron straightline trajectory $\boldsymbol{r}=\boldsymbol{r}_{\mathrm{e}}(t)$.

The availability of TEMs with energy resolution in the $0.1 \mathrm{eV}$ domain has triggered several recent studies of nanoparticle plasmon mapping using EELS. ${ }^{24,25}$ In particular, Fig. 10 shows the near-field characterization of plasmon modes in a gold dumbbell, calculated upon insertion into eqn (9) of the electron-induced electric field obtained from BEM and compared to experiment. ${ }^{25}$ Good qualitative agreement is obtained for the energy position and EELS intensity maps of longitudinal $(l)$ and transverse $(t)$ plasmon maps. The figure shows for comparison calculated near fields for external light excitation, which have similar intensity distributions as EELS, but are not accessible to any existing experimental technique. It is important to stress that a significant EELS intensity is observed and calculated for electrons passing a few nanometres outside the particle, which is due to electromagnetic coupling of the electron to the plasmon modes. This coupling can take place without actual intersection of the electron trajectory with the particle, and thus provides information on the particle while causing minimum damage in the metal.

\section{Surface-enhanced Raman spectroscopy (SERS)}

As mentioned in the introduction, gold nanocrystals hold great promise for biosensing applications because (i) this metal has very limited chemical interaction with biological environments, (ii) it can be easily attached to biomolecules using chemical linkers such as thiols, and (iii) the light intensity can be enormously amplified when the metal is conveniently nanostructured. Intensity enhancement factors of $10^{4}-10^{6}$ have been predicted in the gaps between metallic nanoparticles ${ }^{42}$ or near
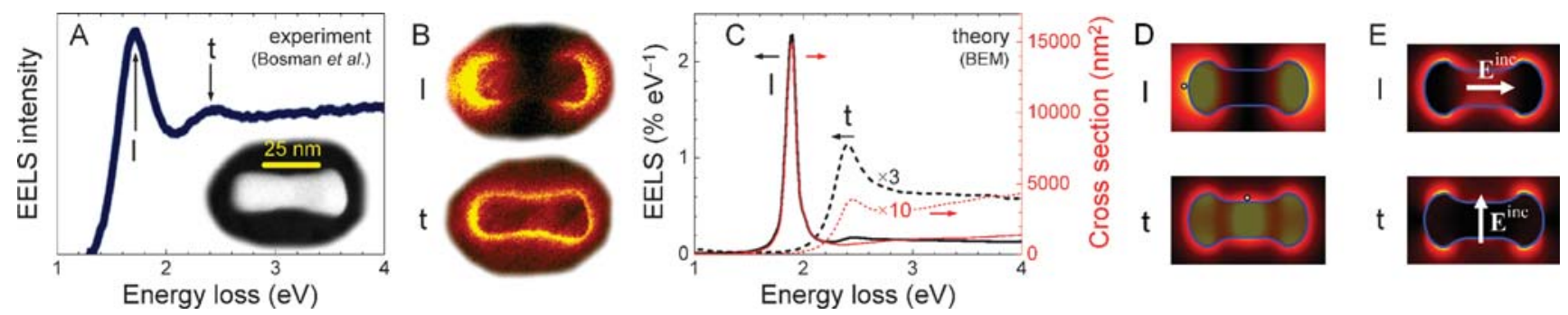

Fig. 10 Probing optical properties of nanoparticles with electrons. (A) Electron energy-loss spectrum (EELS) of a dumbbell gold nanoparticle, taken from ref. 25. A dark-field TEM image of the particle is shown in the inset. (B) Plasmon maps obtained from the intensities of electrons that have lost an energy corresponding to the dipolar longitudinal mode (1.7 eV) and the dipolar transverse mode (2.4 eV), also from ref. 25 . (C) Calculated EELS probability (left scale) and extinction cross section (right scale) of a nanoparticle mimicking the dumbbell of A. The cross section is obtained for external electric field orientations both parallel and perpendicular to the particle axis in order to excite the longitudinal $(l)$ and the transverse $(t)$ plasmon, respectively. The EELS spectra correspond to positions of the electron beam as shown in D by white circles. The longitudinal mode is red-shifted in the experiment, as compared to the calculation, presumably due to the effect of the mica substrate, which is not accounted for here. (D) Calculated EELS plasmon maps, with the particle contour shown as a shaded area. Notice that the experimental images of B exhibit depletion of electron intensity for trajectories passing through the particle because of elastic scattering of the electrons with gold atoms. (E) Near-field plots for longitudinal and transverse polarization of the incident electric field. 
sharp tips. ${ }^{26}$ This enhancement is sufficient to overcome the low, intrinsic, molecular Raman cross section of adsorbed molecules, and results in Raman signals large enough to enable singlemolecule detection.

In a simplified picture, the Raman enhancement in the signal coming from a molecule attached to a nanoparticle is the combined effect of the local increase in light intensity when the particle is illuminated with light of wavelength $\lambda_{\text {in }}$ and the enhancement in the strength of the Raman dipole-emission transition at a longer wavelength $\lambda_{\text {out }}$. The signal is customarily represented as a function of the difference between the inverse of these two wavelengths (i.e., the Raman shift). We can invoke the reciprocity theorem to express the angleaveraged local-field enhancement of the incident light in terms of the enhancement in the emission of a randomly oriented dipole at the same wavelength, $\lambda_{\text {in }}$. This allows us to obtain the ratio between the Raman signal for molecules attached to the particle and the signal in free standing molecules (i.e., the SERS enhancement) as

$$
\mathrm{SERS}=\frac{\int_{\text {surface }} \mathrm{d}^{2} s \Gamma\left(\lambda_{\text {in }}\right) \Gamma\left(\lambda_{\text {out }}\right)}{A \Gamma_{0}\left(\lambda_{\text {in }}\right) \Gamma_{0}\left(\lambda_{\text {out }}\right)},
$$

where the integral is extended over the surface of the nanoparticle in order to average over different positions of the molecule, $A$ is the surface area, $\Gamma(\lambda)$ is the wavelength- and positiondependent emission rate of a dipole at the particle surface, and $\Gamma_{0}(\lambda)$ is the emission rate for the same dipole in air. These rates are easily obtained from the self-induced field on a dipole.

Fig. 11 shows results for SERS enhancement calculated according to eqn (10) for a gold particle with two tips, which mimics the response of actual nanostars. ${ }^{26}$ This has been obtained using BEM for axially-symmetric geometries. ${ }^{15,26}$ Two prominent structures are observed, one at the tip resonance wavelength (above $700 \mathrm{~nm}$ ) and a weaker one at a sphere-like resonance $(500 \mathrm{~nm})$. The former approaches 10 orders of magnitude for molecules absorbed preferentially

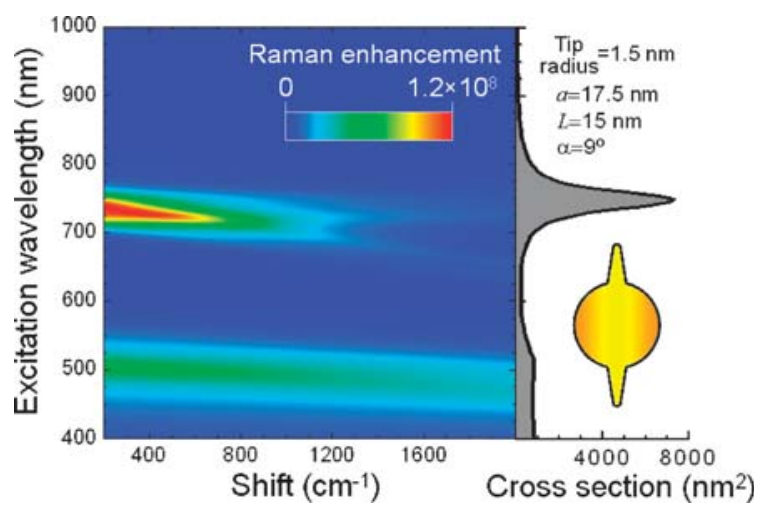

Fig. 11 Left: calculated Raman enhancement in a two-tip gold particle in air. The excitations involved in the Raman process are assumed to be localized $2 \mathrm{~nm}$ away from the gold surface. Right: scattering cross section of the same particle. The particle shape and geometrical parameters are shown in the insets, where $L$ is the tip length, $a$ is the core sphere radius, and $\alpha$ is the semi-angle of aperture of the tip. Reproduced with permission from ref. 26. Copyright 2008, Institute of Physics. near the tip (not shown), which makes these types of particles particularly appealing for SERS studies.

\section{Supported particles}

Validation of the above theoretical models for the shape and size dependence of the surface plasmon resonance lineshape is most readily achieved by comparison with the scattered light of single gold nanoparticles. This can be carried out using darkfield microscopy (DFM), whereby white light is scattered off individual particles dispersed on a microscope slide into a spectrometer. In Fig. 12(A) we show a simplified diagram of the experimental geometry.

Note that the hollow cone of light created as light passes through the dark-field condenser misses the objective, which is only receiving light scattered by the particles. Moreover, surface plasmon modes with a dipole moment in the plane of the substrate dominate this scattering for collection around the normal of the sample slide plane. A typical DFM image of gold nanoparticles as seen through the DFM is shown in Fig. 12(B).

A rigorous comparison of theory and experiment requires measurement of the spectrum and morphology of the same individual nanoparticle, so that a precise correlation of the particle shape and structure with the scattering spectra can be made. ${ }^{20,21}$ Furthermore, the particles are at an interface and it is not possible to define a refractive index for the surrounding medium. For small particles, this can be approximately described by treating them in the electrostatic limit and

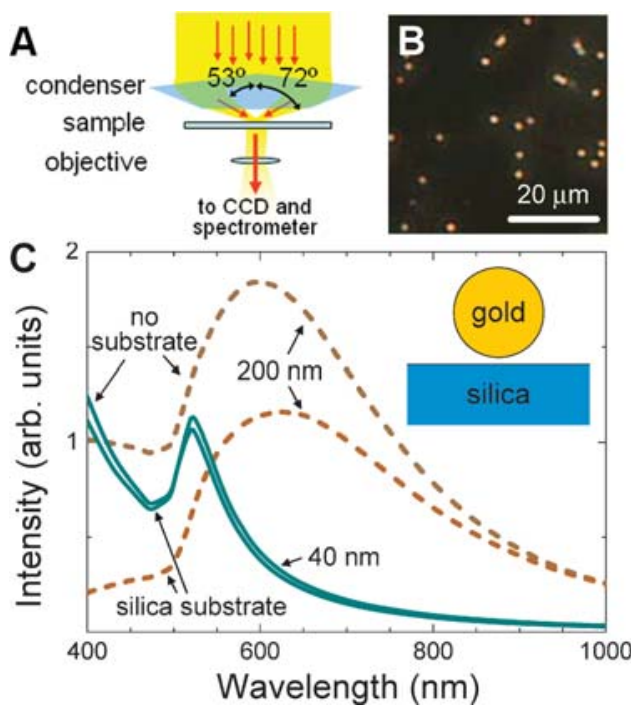

Fig. 12 (A) Illustration of a dark-field microscope. The sample is irradiated by light within a shallow cone of incidence directions. The incident light impinges on the slide but is not collected by the highnumerical-aperture objective. Scattered light however is collected and analyzed by a spectrometer. (B) Dark-field microscope image of individual $200 \mathrm{~nm}$ gold spheres on an ITO-covered glass substrate exposed to air. (C) Scattered spectra calculated with BEM and averaged over the illuminating cone directions for light collection along the sample normal. Gold spheres supported in silica are compared with self-standing particles. The distance between gold and silica surfaces is $1 \mathrm{~nm}$ in the former. Each curve has the colour of the scattered light that it represents for white light illumination. Large spheres undergo larger colour changes. 
calculating the effect of the image dipole on the polarizability of the free particle. The effective polarizability of the ellipsoid of section 2, when placed at a surface-to-surface distance $d$ from a planar substrate with incident electric field parallel to the substrate, is found to be

$$
\alpha_{1}^{\mathrm{eff}}=\frac{\alpha_{1}(1-\beta)}{1-\frac{\alpha_{1} \beta}{32 \pi \varepsilon_{\mathrm{m}}\left(R_{3}+d\right)^{3}}},
$$

where $\alpha_{1}$ is the polarizability along axis 1 parallel to the substrate (see eqn (4)), $\beta=\left(\varepsilon_{\mathrm{s}}-\varepsilon_{\mathrm{m}}\right) /\left(\varepsilon_{\mathrm{s}}+\varepsilon_{\mathrm{m}}\right)$, the axis 3 is taken to be normal to the substrate, and $\varepsilon_{\mathrm{s}}$ is the permittivity of the latter. The modified condition for surface plasmon resonance excitation at contact $(d=0)$ becomes

$$
\varepsilon=-\frac{1-L_{1}+\beta \gamma / 24}{L_{1}-\beta \gamma / 24} \varepsilon_{\mathrm{m}},
$$

where $\gamma=R_{1} R_{2} / R_{3}^{2}$ and $L_{1}$ is the depolarization factor along axis 1 . The major effect of the substrate is to reduce the sensitivity of the surface plasmon resonance to changes in the solvent refractive index. The peak wavelength is either blue- or red-shifted from the homogeneous case, depending on the sign of $\beta$.

Small and large particles with and without a silica substrate are considered in Fig. 12(C), which confirms a larger effect of the substrate for bigger particles. Larger particles require numerical simulation including retardation.

\section{Particle dimers and plasmon chemistry}

Plasmons in composite structures have recently been described in terms of hybridization of plasmon modes supported by the individual parts of those structures, following the methods used to treat electronic orbitals in molecules. Particle dimers constitute a characteristic example of such plasmon structures, ${ }^{43}$ but more complicated geometries have also been shown to be well described using these ideas, for example multi-shell gold particles ${ }^{44}$ and gold nanostars. ${ }^{14}$

Dimers are illustrated in Fig. 13 for spherical particles described by the Drude dielectric function of eqn (2) in the non-retarded limit. The three dipolar modes of each particle are hybridized in the dimer to form plasmon orbitals. Two of them are oriented parallel to the dimer, with a binding plasmon orbital at low energy, which shows a net dipole moment and couples best to external light. Two sets of degenerate modes with dipoles oriented perpendicular to the dimer are also formed. The far-field optical properties of the dimer are governed by the modes with a net dipole. Sphere multipoles become important at very small separations and a singular transition with exotic optical behaviour takes place in the touching limit. ${ }^{45}$ Fully retarded calculations in large neighbouring spheres can be challenging and are best obtained using multiple multipole methods ${ }^{41}$ (see Fig. 9(B)).

Incidentally, the modes of Fig. 13 allow us to obtain the van der Waals interaction energy of the dimer through the zeropoint energy according to

$$
E_{\mathrm{vdW}}=\frac{\hbar}{2} \sum_{j}\left(\omega_{j}-\omega_{j}^{0}\right)
$$

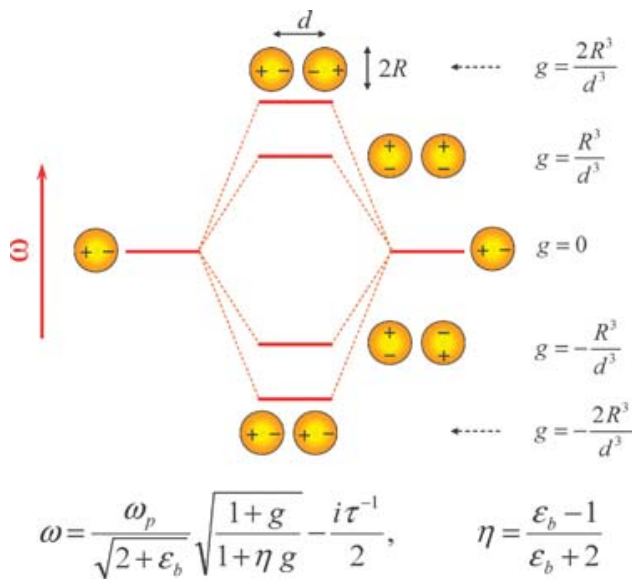

Fig. 13 Plasmon hybridization in interacting nanoparticles. We consider two identical spherical gold nanoparticles of radius $R$ described by the dielectric function of eqn (2). The dipole plasmons of the individual particles are 3-fold degenerate (i.e., the surface plasmon frequency is independent of polarization along $x, y$, and $z$ axes) and have the frequency $\omega_{\mathrm{p}} / \sqrt{2+\varepsilon_{\mathrm{b}}}$, corresponding to the condition $\varepsilon=$ -2 , according to eqn (3) for $l=1$ and particles in $\operatorname{air}\left(\varepsilon_{\mathrm{m}}=1\right)$. The modes of the interacting system are derived from the eigenfrequencies of the two-particle DDA equations. The mode frequencies respond to the formula quoted at the bottom, with values of $g$ shown in the insets and containing the dependence on centre-to-centre separation, $d$. Retardation effects and higher-order multipoles have been neglected in these expressions.

where the sum is extended over mode frequencies of the interacting and the non-interacting particles, $\omega_{j}$ and $\omega_{j}^{0}$, respectively. The dipole modes of Fig. 13 provide the dominant contribution to the sum of eqn (11) when the separation is large compared to the radius $(d \gg R)$. We find

$$
E_{\mathrm{vdW}} \approx \frac{3 \hbar \omega_{\mathrm{p}}}{4 \sqrt{2+\varepsilon_{\mathrm{b}}}}(3 \eta+1)(\eta-1)\left(\frac{R}{d}\right)^{6},
$$

which yields the expected non-retarded $1 / d^{6}$ dependence for the van der Waals energy. Here, $\eta=\left(\varepsilon_{\mathrm{b}}-1\right) /\left(\varepsilon_{\mathrm{b}}+2\right)$.

\section{Particle arrays}

\subsection{Effective medium theories}

Particle arrays consisting of nanoparticles embedded in a homogeneous matrix present effective properties which are not only a function of the component materials and the filling fraction, but also depend on the particle shape and spatial distribution. Although Mie theory describes light absorption and scattering in dilute dispersions of weakly-interacting spheres relatively well, single-particle approaches fail in general to interpret the interactions in particle arrays and theoretical effective-medium models are required. One of the first and most enduring models to describe the permittivity of random composites is the Maxwell-Garnett approximation. ${ }^{2}$ This model derives from the Clausius-Mossotti formula, ${ }^{27}$ which links the polarizability $\alpha$ of the small particles in an array to the effective dielectric function of such material $\varepsilon_{\mathrm{eff}}$ 
according to

$$
\frac{\varepsilon_{\mathrm{eff}}-\varepsilon_{\mathrm{m}}}{\varepsilon_{\mathrm{eff}}+2 \varepsilon_{\mathrm{m}}}=\frac{\alpha}{3 v \varepsilon_{\mathrm{m}}}
$$

where $v$ is the average volume per particle. This formula does not take into consideration non-dipolar interactions, and the effect of local order is neglected. However, it works quite well when applied to dilute samples, for which only dipolar interactions are important. Maxwell-Garnett theory arises from inserting eqn (3) with $l=1$ into the Clausius-Mossotti formula:

$$
\varepsilon_{\mathrm{eff}}=\varepsilon_{\mathrm{m}} \frac{\varepsilon(1+2 f)+2 \varepsilon_{\mathrm{m}}(1-f)}{\varepsilon(1-f)+\varepsilon_{\mathrm{m}}(2+f)},
$$

where $f$ is the filling fraction of gold (described by $\varepsilon$ ) inside the matrix (described by $\varepsilon_{\mathrm{m}}$ ).

Maxwell-Garnett theory was found to explain the optical properties of composites formed by silica-coated gold particles very well up to volume fractions of at least $f=0.3$. $^{46}$ The volume fraction was regulated by varying the silica shell thickness.

When the particles are closer together, higher-order multipoles beyond the dipole can be relevant. Effective dielectric functions can still be defined, but their calculation becomes more involved, although quasi-analytical solutions to this problem have been postulated. ${ }^{47}$

\subsection{Planar particle arrays}

van der Waals interactions can produce self-assembly of nanoparticles deposited on a planar substrate, in particular through the layer-by-layer deposition method, which has been utilized to achieve ordered multilayer arrays. Accordingly, a layer-by-layer method has been envisaged to simulate the optical response of these structures, relying on a multipolar expansion of the electromagnetic field about each particle, followed by subsequent multiple scattering within layers in a multipole basis set and between layers in a plane-wave basis set. ${ }^{48}$

For finite arrays of particles with some degree of disorder, the generalized multipole expansion provides an efficient way of solving Maxwell's equations rigorously in the presence of up to several thousand particles arranged in completely arbitrary positions. ${ }^{41}$ The power of this method for the simplest case of a dimer has already been illustrated in Fig. 9(B), and more complicated arrangements of gold nanoparticles have also been investigated. ${ }^{49}$

\section{Summary and outlook}

We have presented a compendium of the most relevant methods used to model the optical response of gold nanoparticles. Several numerical procedures are well suited for predictive simulations of optical spectra, and in particular the advantages and disadvantages of BEM, DDA and FDTD have been discussed thoroughly. These methods yield the solution to Maxwell's equations when gold and its environment are described by frequency-dependent dielectric functions, but non-local effects can be important both for small particle separations and for particles below $10 \mathrm{~nm}$ in size. Although a phenomenological recipe exists that yields good agreement with experiment for spherical particles (see Fig. 6), a generalization of the simulation methods discussed here to include non-local effects in arbitrarilyshaped particles is still needed. In this direction, experiment is currently progressing ahead of theory, and in particular a detailed report has been given on the relative partial contribution of non-local effects (and specifically surface-scattering), radiative losses, and pure bulk absorption to plasmon-line broadening in gold and silver particles. ${ }^{17}$

A qualitative description of plasmon resonances in metallic nanoparticles is generally possible using analytical formulae and tabulated data like those presented in sections 2 and 3 (without and with retardation, respectively) for spheres, ellipsoids, and nanorods. Accurate simulations for more complicated shapes must rely on numerical methods. Examples of the application of these methods to calculate far-field extinction spectra (see Fig. 7-9 and 12) and near-field information encoded in electron energy-loss spectra (see Fig. 10) have been offered. We hope that this review can help to coordinate the experimental and theoretical work currently underway in the field of metal nanoparticle optics and spectroscopy.

\section{Acknowledgements}

This work was supported by the Spanish MEC (NAN200408843-C03/05, MAT2007-66050, and MAT2007-62696) and Xunta de Galicia. PM acknowledges support through ARC Federation Fellowship FF0561486 and DP Grant 0451651. JR-F acknowledges an FPU scholarship.

\section{References}

1 L. B. Hunt, Gold Bull., 1976, 9, 134.

2 J. C. Maxwell-Garnett, Philos. Trans. R. Soc. London, Ser. A, 1904, 203, 385; J. C. Maxwell-Garnett, Philos. Trans. R. Soc. London, Ser. A, 1906, 205, 237.

3 G. Mie, Ann. Phys. (Leipzig), 1908, 25, 377.

4 R. Gans, Ann. Phys. (Leipzig), 1912, 37, 881.

5 D. C. Skillman and C. R. Berry, J. Chem. Phys., 1968, 48, 3297.

6 E. M. Purcell and C. R. Pennypacker, Astrophys. J., 1973, 186, 705.

7 B. T. Draine and P. J. Flatau, J. Opt. Soc. Am. A, 1994, 11, 1491.

8 B. T. Draine and P. J. Flatau, 2004, http://arxiv.org/abs/astro-ph/ $0409262 \mathrm{v} 2$.

9 K. L. Kelly, E. Coronado, L. L. Zhao and G. C. Schatz, J. Phys. Chem. B, 2003, 107, 668.

10 L. M. Liz-Marzán, Langmuir, 2006, 22, 32.

11 M. P. Pileni, J. Phys. Chem. C, 2007, 111, 9019.

12 C. Noguez, J. Phys. Chem. C, 2007, 111, 3806.

13 A. Taflove, Computational Electrodynamics: The Finite-Difference Time-Domain Method, Artech House, Boston, 1995.

14 F. Hao, C. L. Nehl, J. H. Hafner and P. Nordlander, Nano Lett., 2007, 7, 729.

15 F. J. García de Abajo and A. Howie, Phys. Rev. Lett., 1998, 80, 5180; F. J. García de Abajo and A. Howie, Phys. Rev. B: Condens. Matter Mater. Phys., 2002, 65, 115418.

16 V. Myroshnychenko, E. Carbó-Argibay, I. Pastoriza-Santos, J. Pérez-Juste, L. M. Liz-Marzán and F. J. García de Abajo, $A d v$. Mater., DOI: 10.1002/adma.200703214.

17 M. Hu, C. Novo, A. Funston, H. Wang, H. Petrova, S. Zou, P. Mulvaney, Y. Xia and G. V. Hartland, J. Mater. Chem., 2008, 18, 1949.

18 R. H. Ritchie, Phys. Rev., 1957, 106, 874.

19 H. C. van de Hulst, Light Scattering by Small Particles, Dover, New York, 1981.

20 J. J. Mock, M. Barbic, D. R. Smith, D. A. Schultz and S. Schultz, J. Chem. Phys., 2002, 116, 6755.

21 C. Novo, A. M. Funston, I. Pastoriza-Santos, L. M. Liz-Marzán and P. Mulvaney, Angew. Chem., Int. Ed., 2007, 46, 3517. 
22 C. Chicanne, T. David, R. Quidant, J. C. Weeber, Y. Lacroute, E. Bourillot, A. Dereux, G. Colas des Francs and C. Girard, Phys. Rev. Lett., 2002, 88, 097402.

23 N. Yamamoto, K. Araya and F. J. García de Abajo, Phys. Rev. B: Condens. Matter Mater. Phys., 2001, 64, 205419.

24 J. Nelayah, M. Kociak, O. Stéphan, F. J. García de Abajo, M. Tencé, L. Henrard, D. Taverna, I. Pastoriza-Santos, L. M. LizMarzán and C. Colliex, Nat. Phys., 2007, 3, 348.

25 M. Bosman, V. J. Keast, M. Watanabe, A. I. Maaroof and M. B. Cortie, Nanotechnology, 2007, 18, 165505.

26 P. S. Kumar, I. Pastoriza-Santos, B. Rodríguez-González, F. J. García de Abajo and L. M. Liz-Marzán, Nanotechnology, 2008, 19, 015606.

27 J. D. Jackson, Classical Electrodynamics, Wiley, New York, 1999.

28 P. B. Johnson and R. W. Christy, Phys. Rev. B: Solid State, 1972, 6, 4370.

29 R. Ruppin, Phys. Status Solidi B, 1978, 87, 619.

30 H. Kuwata, H. Tamaru, K. Esumi and K. Miyano, Appl. Phys. Lett., 2003, 83, 4625.

31 U. Kreibig and M. Vollmer, Optical Properties of Metal Clusters, Springer-Verlag, Berlin, 1995

32 E. Carbó-Argibay, B. Rodríguez-González, J. Pacifico, I. Pastoriza-Santos, J. Pérez-Juste and L. M. Liz-Marzán, Angew. Chem., Int. Ed., 2007, 46, 8983.

33 B. T. Draine and J. Goodman, Astrophys. J., 1993, 405, 685.

34 J. J. Goodman, B. T. Draine and P. J. Flatau, Opt. Lett., 1991, 16, 1198.

35 S. Prescott and P. Mulvaney, J. Appl. Phys., 2006, 99, 123504.
36 G. Colas des Francs, C. Girard, J. C. Weeber, C. Chicane, T. David, A. Dereux and D. Peyrade, Phys. Rev. Lett., 2001, 86 4950.

37 I. Pastoriza-Santos, A. Sánchez-Iglesias, F. J. García de Abajo and L. M. Liz-Marzán, Adv. Funct. Mater., 2007, 17, 1443.

38 K. S. Yee, IEEE Trans. Antennas Propag., 1966, AP-14, 302.

39 K. Busch and S. John, Phys. Rev. E: Stat. Phys., Plasmas, Fluids, Relat. Interdiscip. Top., 1998, 58, 3896.

40 F. Ouyang and M. Isaacson, Ultramicroscopy, 1989, 31, 245.

41 F. J. García de Abajo, Phys. Rev. Lett., 1999, 82, 2776; F. J. García de Abajo, Phys. Rev. B: Condens. Matter Mater. Phys., 1999, 60, 6086.

42 H. Xu, E. J. Bjerneld, M. Käll and L. Börjesson, Phys. Rev. Lett., 1999, 83, 4357.

43 P. Nordlander, C. Oubre, E. Prodan, K. Li and M. I. Stockman, Nano Lett., 2004, 4, 899.

44 E. Prodan, C. Radloff, N. J. Halas and P. Nordlander, Science, 2003, 302, 419.

45 I. Romero, J. Aizpurua, G. W. Bryant and F. J. García de Abajo, Opt. Express, 2006, 14, 9988.

46 T. Ung, L. M. Liz-Marzán and P. Mulvaney, J. Phys. Chem. B, 2001, 105, 3441.

47 D. R. McKenzie and R. C. McPhedran, Nature, 1977, 265 128.

48 N. Stefanou, V. Yannopapas and A. Modinos, Comput. Phys. Commun., 2000, 132, 189.

49 S. Bidault, F. J. García de Abajo and A. Polman, J. Am. Chem. Soc., 2008, 130, 2750. 